\title{
TENDERIZACIÓN POST-MORTEM DE LA CARNE DE LOS DISTIN- TOSBIOTIPOS AMPARADOS POR LA IGP TERNERA ASTURIANA
}

\author{
POST-MORTEM TENDERIZATION OF BEEF FROM DIFFERENT BIOTYPES INCLUDED \\ IN THE PGI TERNERA ASTURIANA
}

\author{
Sierra, V. ${ }^{1}, 2$, Fernández-Suárez, V. ${ }^{1}$, Castro, P. ${ }^{1}$, Osoro, K. ${ }^{1}$, Rodríguez-Colunga, M.J. ${ }^{2}$, \\ Vega-Naredo, I. ${ }^{2}$, García- Macía, M. ${ }^{2}$, Coto-Montes, A. ${ }^{2}$ y Oliván, M. ${ }^{*}$
}

\begin{abstract}
†Área de Sistemas de Producción Animal. SERIDA. Villaviciosa. Asturias. España. *mcolivan@serida.org ${ }^{2}$ Departamento de Morfología y Biología Celular. Facultad de Medicina. Universidad de Oviedo. Oviedo. Asturias. España.
\end{abstract}

\section{Palabras clave adicionales}

Raza. Hipertrofia muscular. Dureza. Calpaínas. Proteolisis.

\section{RESUMEN}

Se estudió la actividad de la $\mu$-calpaína y la tenderización a lo largo de la maduración postmortem (de $2 \mathrm{~h}$ a $21 \mathrm{~d}$ ) en carne procedente de distintos biotipos amparados por la Indicación Geográfica Protegida (IGP) Ternera Asturiana, según la raza (Asturiana de los Valles, AV y Asturiana de la Montaña, AM) y el genotipo para la mutación en el gen de la miostatina causante de la hipertrofia muscular $(m h / m h, m h /+,+/+)$. Esta mutación produjo diferencias significativas en la evolución post-mortem de la calidad de la carne promoviendo un descenso más rápido del pH y una activación más temprana de las calpaínas y por tanto de la proteolisis en los biotipos AV $(\mathrm{mh} / \mathrm{mh}$ y $m h /+$ ) que mostraron un ritmo de tenderización más temprano que la carne de los biotipos normales $(+/+)$ de ambas razas. Los resultados indican que las diferencias encontradas entre biotipos en $\mathrm{pH}$ y actividad de $\mu$-calpaína muestran un paralelismo con el proceso de tenderización.

\section{SUMMARY}

Tenderization and $\mu$-calpain activity were studied along post-mortem maduration ( $2 \mathrm{~h}$ to $21 \mathrm{~d}$ ) on beef from different biotypes included in the Protected Geographical Indication (PGI) Ternera Asturiana attending to breed (Asturiana de los Valles, AV and Asturiana de la Montaña, AM) and

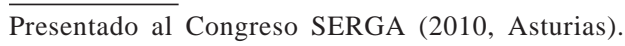

\section{AdDitiOnAL KEYWORDS}

Breed. Muscular hypertrophy. Toughness. Calpains. Proteolysis.

genotype for myostatine mutation causing muscular hypertrophy $(m h / m h, m h /+$ and $+/+)$. This mutation produced significant differences in meat quality, promoting a faster $\mathrm{pH}$ decline and earlier activation of calpains, which resulted in faster proteolysis in $\mathrm{AV}(\mathrm{mh} / \mathrm{mh}$ and $\mathrm{mh} /+)$ biotypes, that showed earlier tenderization rate than normal $(+/+)$ biotypes of both breeds. The results showed that differences among biotypes in $\mathrm{pH}$ and $\mu$ calpain activity paralleled the tenderization process.

\section{INTRODUCCIÓN}

La terneza es un parámetro de calidad muy importante en la carne de vacuno, que dependerá en parte de la acción de distintos sistemas proteolíticos sobre proteínas estructurales clave. Diversos estudios apuntan a la $\mu$-calpaína como la principal responsable de la tenderización post-mortem de la carne (Koohmaraie y Geesink, 2006).

Asturias se distingue por la producción de carne de calidad, amparada por la Indicación Geográfica Protegida (IGP) Ternera Asturiana, que engloba distintos productos de las razas autóctonas Asturiana de los Valles (AV), Asturiana de la Montaña (AM) y sus cruces, además de diferentes biotipos 
de la raza AV $(m h / m h, m h /+\mathrm{y}+/+)$ dependiendo de la presencia de una mutación en el gen de la miostatina que genera hipertrofia muscular. En general, la carne de los genotipos $m h$ de la raza AV es más clara, con menor contenido graso y mayores pérdidas de jugo (Aldai et al., 2006), presentando además una tenderización post-mortem más rápida (Sierra et al., 2010), mientras que la carne de genotipos normales (+/+) de ambas razas (AV y AM) tiene mayor contenido de pigmentos y grasa intramuscular y un patrón de tenderización más lento, por lo que requiere mayor tiempo de maduración para alcanzar una calidad sensorial óptima (Sierra et al., 2010). El objetivo de este estudio es analizar la actividad de la $\mu$-calpaína y su influencia en el proceso de tenderización de la carne en los distintos biotipos amparados por la IGP Ternera Asturiana.

\section{MATERIALES Y MÉTODOS}

Se utilizaron 16 terneros añojos machos de las razas AM y AV y distintos biotipos con respecto a la hipertrofia muscular $(\mathrm{mh} /$ $m h, m h /+,+/+)$ incluyendo 4 animales por categoría. Los animales (alimentados en intensivo) fueron sacrificados a los $20 \mathrm{me}$ ses de edad y aproximadamente $600 \mathrm{~kg}$ de peso vivo. Se tomaron muestras de $20 \mathrm{~g}$ del Longissimus dorsi (LD) en la canal a las 2, 12 y 24 h post-mortem, para el análisis de la actividad de $\mu$-calpaína, que se congelaron en nitrógeno líquido y se almacenaron a $-80^{\circ} \mathrm{C}$. Al mismo tiempo se registraron la temperatura y el $\mathrm{pH}$. A las $24 \mathrm{~h}$ post-mortem, se extrajo el LD y se dividió en filetes que se envasaron al vacío y se mantuvieron en refrigeración a lo largo de la maduración (48 h, 3 d, 7 d, 14 d y 21 d) hasta la realización de los análisis correspondientes. La actividad de $\mu$-calpaína (nativa y autolisada) se analizó por zimografía, siguiendo el método de Veiseth et al. (2001). Los geles se cuantificaron utilizando el software QuantityOne (Biorad) según Pomponio et al. (2010). La dureza instrumental se midió en carne cocinada y se registró como resistencia al corte $(\mathrm{kg})$ en una máquina TA.XT Plus utilizando una cuchilla Warner-Bratzler.

\section{RESULTADOSYDISCUSIÓN}

$\mathrm{El} \mathrm{pH}$ registrado a las $2 \mathrm{~h}$ post-mortem fue significativamente $(\mathrm{p}<0,05)$ más bajo en la carne de los terneros AV $(\mathrm{mh} / \mathrm{mh})$, lo que parece indicar un metabolismo muscular más glicolítico. El biotipo también afectó al proceso de tenderización (tabla I), de modo que

Tabla I. Efecto del biotipo (en líneas) y del tiempo de maduración (en columnas) sobre la dureza instrumental (WB, en $\mathrm{kg}$ ). (Effect of biotype (in rows) and ageing time (in columns) on meat toughness (WB, in $\mathrm{kg})$ ).

\begin{tabular}{cccccc}
\hline Maduración (días) & $\mathrm{AV}(m h / m h)$ & $\begin{array}{c}\text { Biotipo } \\
\mathrm{AV}(m h /+)\end{array}$ & $\mathrm{AV}(+/+)$ & $\mathrm{AM}$ & Significación \\
\hline 3 & $6,92^{\mathrm{b}}$ & $7,50^{\mathrm{b}}$ & $8,66^{\mathrm{b}}$ & $8,47^{\mathrm{c}}$ & $\mathrm{NS}$ \\
7 & $5,99^{\mathrm{ABb}}$ & $4,78^{\mathrm{Aa}}$ & $8,29^{\mathrm{Cb}}$ & $7,59^{\mathrm{BCbc}}$ & N* \\
14 & $5,16^{\mathrm{a}}$ & $4,93^{\mathrm{a}}$ & $6,82^{\mathrm{ab}}$ & $5,57^{\mathrm{a}}$ & $\mathrm{NS}$ \\
21 & $5,549^{\mathrm{a}}$ & $4,55^{\mathrm{a}}$ & $5,18^{\mathrm{a}}$ & $5,96^{\mathrm{ab}}$ & NS \\
Significación & $* * *$ & $* *$ & $*^{*}$ & &
\end{tabular}

Para cada tiempo de maduración, medias en la misma línea seguidas por letras mayúsculas distintas son significativamente diferentes para $\mathrm{p}<0,05$.

Para cada biotipo, medias en la misma columna seguidas por letras minúsculas distintas son significativamente diferentes para $p<0,05$. 


\section{TENDERIZACIÓN DE LA CARNE DE TERNERA ASTURIANA SEGÚN EL BIOTIPO}

la carne de los biotipos $m h$ alcanzó los valores más bajos de dureza WB a los 7 días post-mortem, lo que indica una tenderización más temprana en los biotipos con hipertrofia muscular, coincidiendo con resultados previos (Oliván et al., 2004; Sierra et al., 2010).

En cuanto a la actividad de la $\mu$-calpaína (tanto nativa como autolisada) (tabla II), se observó un descenso significativo $(\mathrm{p}<0,05)$ a lo largo de la maduración en todos los biotipos, ocurriendo más tempranamente en la carne de los biotipos $m h(48 \mathrm{~h})$ que en los normales de ambas razas ( $7 \mathrm{~d})$.

También se encontraron diferencias significativas en la actividad de $\mu$-calpaína entre biotipos. Así, la carne de los animales AM mostró la mayor actividad de $\mu$-calpaína nativa en el post-mortem temprano ( $2 \mathrm{~h}-3 \mathrm{~d})$ junto con la de los terneros AV $(\mathrm{mh} / \mathrm{mh})$ ( $2 \mathrm{~h}-24 \mathrm{~h}$ ), mostrando estos dos biotipos un patrón de actividad de $\mu$-calpaína nativa muy similar, con un fuerte descenso de actividad (>90\%) a lo largo de la maduración, que ocurrió de forma más temprana en el biotipo AV $(\mathrm{mh} / \mathrm{mh})(12 \mathrm{~h}-48 \mathrm{~h})$ que en el AM (3 h-7 d). Sin embargo, los biotipos heterocigoto $(\mathrm{mh} /+)$ y normal $(+/+)$ de la raza AV mostraron actividades iniciales de $\mu$-calpaína menores y un descenso de actividad más reducido y gradual $(70 \%)$. Por otro lado, la carne del biotipo AV $(+/+)$

Tabla II. Efecto del biotipo (en líneas) y el tiempo de maduración (en columnas) sobre la actividad de las formas nativa y autolisada de la $\mu$-calpaína. (Effect of biotype (in rows) and ageing time (in columns) on native $\mu$-calpain and autolyzed $\mu$-calpain activities).

\begin{tabular}{|c|c|c|c|c|c|c|}
\hline & Maduración & $\mathrm{AV}(\mathrm{mh} / \mathrm{mh})$ & $\begin{array}{c}\text { Biotipo } \\
\text { AV }(\mathrm{mh} /+)\end{array}$ & $\mathrm{AV}(+/+)$ & AM & Significación \\
\hline \multicolumn{7}{|l|}{$\mu$ - calpaína nativa } \\
\hline & $2 \mathrm{~h}$ & $0,96^{\mathrm{ABb}}$ & $0,68^{\mathrm{ABbc}}$ & $0,51^{\mathrm{A}}$ & $1,04^{\mathrm{Bb}}$ & * \\
\hline & $12 \mathrm{~h}$ & $0,92^{\mathrm{b}}$ & $0,71^{\mathrm{c}}$ & 0,70 & $0,93^{\mathrm{b}}$ & NS \\
\hline & $24 \mathrm{~h}$ & $0,85^{\mathrm{ab}}$ & $0,78^{c}$ & 0,74 & $1,00^{\mathrm{b}}$ & NS \\
\hline & $48 \mathrm{~h}$ & $0,21^{\mathrm{Aa}}$ & $0,67^{\mathrm{ABbc}}$ & $0,60^{A}$ & $1,06^{\mathrm{Bb}}$ & $* *$ \\
\hline & $3 d$ & $0,12^{\mathrm{Aa}}$ & $0,46^{\mathrm{ABabc}}$ & $0,53^{A B}$ & $0,93^{\mathrm{Bb}}$ & $* *$ \\
\hline & $7 \mathrm{~d}$ & $0,15^{\mathrm{a}}$ & $0,26^{\mathrm{ab}}$ & 0,26 & $0,22^{\mathrm{a}}$ & NS \\
\hline & $14 d$ & $0,15^{a}$ & $0,27^{\mathrm{ab}}$ & 0,17 & $0,10^{\mathrm{a}}$ & NS \\
\hline & $21 \mathrm{~d}$ & $0,08^{a}$ & $0,22^{a}$ & 0,16 & $0,09^{a}$ & NS \\
\hline & Sign. & $\star \star \star *$ & 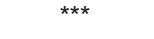 & * & 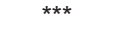 & \\
\hline \multicolumn{7}{|c|}{ «- calpaína autolisada } \\
\hline & $2 \mathrm{~h}$ & $1,34^{b}$ & $1,92^{b}$ & $2,29^{c}$ & $1,88^{b}$ & NS \\
\hline & $12 \mathrm{~h}$ & $1,30^{\mathrm{Ab}}$ & $1,58^{\mathrm{ABb}}$ & $1,97^{\mathrm{Bbc}}$ & $1,78 A^{\mathrm{Bb}}$ & * \\
\hline & $24 \mathrm{~h}$ & $1,00^{A b}$ & $1,03^{\mathrm{Aab}}$ & $2,02^{\mathrm{Bbc}}$ & $1,66 \mathrm{~A}^{\mathrm{Bb}}$ & * \\
\hline & $48 \mathrm{~h}$ & $0,25^{\text {Аа }}$ & $0,55^{\mathrm{ABa}}$ & $1,37^{\mathrm{Cbc}}$ & $1,18 \mathrm{~B}^{\mathrm{Cb}}$ & $\star *$ \\
\hline & $3 d$ & $0,16^{a}$ & $0,39^{a}$ & $1,03^{\mathrm{ab}}$ & $0,94^{\mathrm{ab}}$ & NS \\
\hline & $7 d$ & $0,12^{a}$ & $0,20^{a}$ & $0,23^{a}$ & $0,28^{a}$ & NS \\
\hline & $14 d$ & $0,13^{a}$ & $0,19^{a}$ & $0,12^{\mathrm{a}}$ & $0,12^{\mathrm{a}}$ & NS \\
\hline & $21 d$ & $0,07^{\mathrm{Aa}}$ & $0,17^{\mathrm{Ba}}$ & $0,14^{\mathrm{ABa}}$ & $0,06^{\mathrm{Aa}}$ & $* *$ \\
\hline & Sign. & $* * *$ & $* * *$ & $* * *$ & 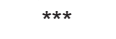 & \\
\hline
\end{tabular}

Para cada tiempo de maduración, medias en la misma línea seguidas por letras mayúsculas distintas son significativamente diferentes para $p<0,05$.

Para cada biotipo, medias en la misma columna seguidas por letras minúsculas distintas son significativamente diferentes para $p<0,05$. 


\section{SIERRA ET AL.}

presentó niveles de actividad de $\mu$-calpaína autolisada significativamente mayores $(\mathrm{p}<0,05)$ que la del biotipo AV $(\mathrm{mh} / \mathrm{mh}) . \mathrm{Al}$ considerar la relación entre las calpaínas y la dureza instrumental, se encontró una correlación positiva $(\mathrm{p}<0,01)$ entre la actividad de la $\mu$-calpaína autolisada a tiempos tempranos post-mortem (12 h-24 h) y la dureza instrumental de la carne a los 7 días de maduración $(r>0,6)$.

\section{CONCLUSIONES}

La carne de los distintos biotipos amparados por la IGP Ternera Asturiana mostró un proceso de tenderización similar pero con distinta escala temporal.

El músculo LD de los biotipos $m h$ presentó un pH más bajo, niveles más altos de actividad de $\mu$-calpaína en tiempos cortos de maduración y un descenso más rápido de la misma, lo que redunda en una tenderización más temprana de la carne. Por el

\section{BIBLIOGRAFÍA}

Aldai, N., Murray, B.E., Oliván, M., Martínez, A., Troy, D.J., Osoro, K. and Nájera, A.I. 2006. The influence of breed and mh-genotype on carcass conformation, meat physico-chemical characteristics, and the fatty acid profile of muscle from yearling bulls. Meat Sci., 72: 486-495.

Koohmaraie, M. and Geesink, G.H. 2006. Contribution of post-mortem muscle biochemistry to the delivery of consistent meat quality with particular focus on the calpain system. Meat Sci., 74: 34-43.

Oliván, M., Martínez, A., Osoro, K., Sañudo, C., Panea, P., Olleta, J.L., Campo, M.M., Oliver, M.A., Serra, X., Gil, M. and Piedrafita, J. 2004. Effect of muscular hypertrophy on physicochemical, biochemical and texture traits of meat contrario, la carne de los biotipos normales de ambas razas siguió un proceso de tenderización más lento pero gradual, probablemente debido a que el tejido muscular mantuvo durante más tiempo la actividad de la $\mu$-calpaína. Los cambios post-mortem observados en $\mu$-calpaína mostraron paralelismo con la evolución de la dureza de la carne, estando altamente correlacionados, lo que corrobora el papel relevante de las calpaínas en el proceso de tenderización de la carne.

\section{AGRADECIMIENTOS}

Los autores agradecen a M.J. García y M.J. Martínez su colaboración en los experimentos. Este estudio ha sido financiado por el INIA a través del proyecto RTA200700087-C02 y fondos FEDER. V. Sierra agradece al INIA su beca predoctoral e I. VegaNaredo agradece al Ministerio de Ciencia e Innovación su beca FPU.

from yearling bulls. Meat Sci., 68: 567-575.

Pomponio, L., Ertbjerg, P., Karlsson, A.H., Costa, L.N. and Lametsch, R. 2010. Influence of early $\mathrm{pH}$ decline on calpain activity in porcine muscle. Meat Sci., 85: 110-114.

Sierra, V., Guerrero, L., Fernández-Suárez, V. Martínez, A., Castro, P., Osoro, K. RodríguezColunga, M.J., Coto-Montes, A. and Oliván, M. 2010. Eating quality of beef from biotypes included in the PGI Ternera Asturiana showing distinct physicochemical characteristics and tenderization pattern. Meat Sci., 86: 343-351.

Veiseth, E., Shackelford, S.D., Wheeler, T.L. and Koohmaraie, M. 2001. Effect of post-mortem storage on $\mu$-calpain and $\mu$-calpain in ovine skeletal muscle. J. Anim. Sci., 70: 3035-3043. 